\title{
Trap-Assisted Dopant Compensation Prevents Shunting in poly-Si Passivating Interdigitated Back Contact Silicon Solar Cells
}

Matthew B. Hartenstein, ${ }^{1,2}$ Caleb Stetson ${ }^{1,2}$, William Nemeth, ${ }^{2}$ Vincenzo LaSalvia, ${ }^{2}$ Steven P. Harvey, ${ }^{2}$ San Theingi, ${ }^{2}$ Matthew Page, ${ }^{2}$ Chun-Sheng Jiang, ${ }^{2}$ Mowafawk M. Al-Jassim, ${ }^{2}$ David L. Young, ${ }^{2}$ Sumit Agarwal ${ }^{1,2^{*}}$, and Paul Stradins ${ }^{1,2, *}$.

${ }^{1}$ Colorado School of Mines, Golden, CO, 80401, USA, ${ }^{2}$ National Renewable Energy Laboratory, Golden,

\section{Supplementary Information}

$\mathrm{CO}, 80401, \mathrm{USA}$.

\section{Morphology of sample measured by scanning spreading resistance microscopy (SSRM)}

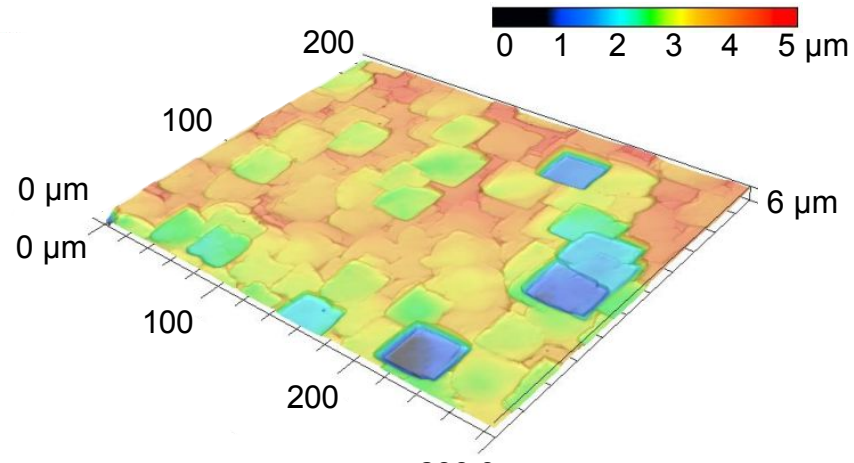

286.3

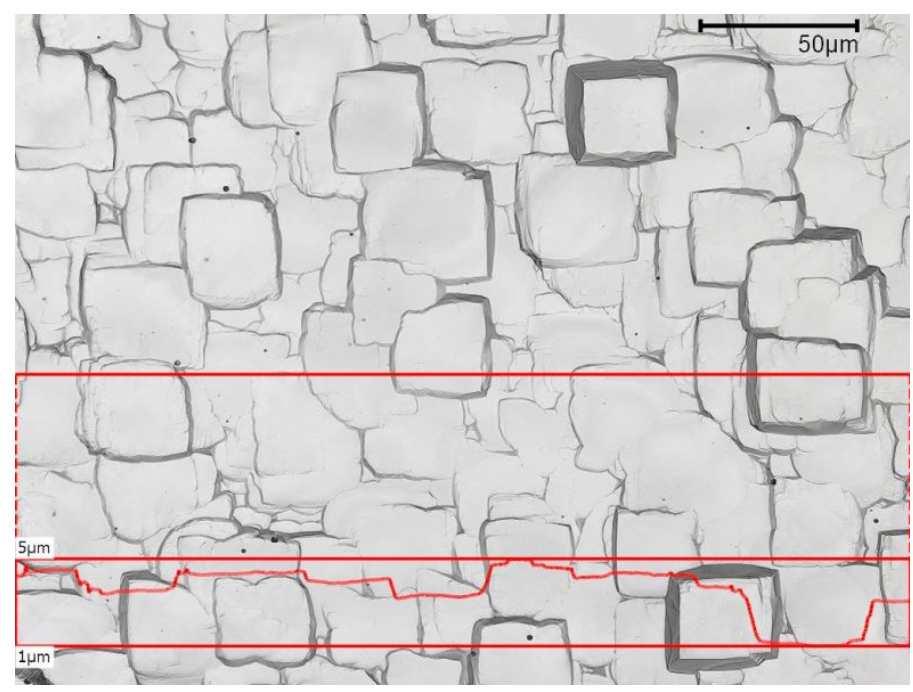

Figure S1 Three-dimensional (top) map of the surface topology of the $\sim 300 \times 200 \mu \mathrm{m}$ region of a planarized crystalline $\mathrm{Si}$ wafer scanned by laser microscopy, which is representative of the sample surfaces measured with SSRM. (Bottom) Optical microscope image of the same region showing inverted $\sim 30 \mu \mathrm{m}$ wide mesas. The height variation along the line scan for top red line is shown in the red box underneath. The out of plane height variation for this line scan is over 1-5 $\mu \mathrm{m}$ over the $\sim 300 \mu \mathrm{m}$ width. The morphology of this sample surface is obtained following $\mathrm{KOH}$ etching of saw damaged crystalline $\mathrm{Si}(c-\mathrm{Si})$ wafers. Because of this roughness, to avoid damaging the probe tip, a lower probe force, 1-2 $\mu \mathrm{N}$, was used for the SSRM measurements compared to typical SSRM probe forces of $\sim 10 \mu \mathrm{N}$ on atomically flat samples. This resulted in an overestimation of the local resistivity of the sample. 


\section{Resistor simulation details}

Carrier transport in the contaminated isolation region between the $p$-and $n$-type fingers is a three-dimensional problem, but for simplicity we conducted one-dimensional calculations assuming uniform dopant concentration throughout the thickness of the poly-Si film. For a high shunt resistance, the $p-i-n$ structure can most simply be modelled as a resistor, considering drift currents from applied bias, but not diffusion current, space charge effects, or emission of carriers from trap states. Dopant profiles input into the simulation were based on lateral TOF-SIMS data or assumed to be an error function spreading from the edge of the mask defining the intentionally doped fingers. From previous experiments, we find that $\sim 10 \%$ of boron atoms and $\sim 30 \%$ of phosphorus atoms are active for total dopant concentrations in poly-Si of $10^{20}-10^{21} \mathrm{~cm}^{-3}$ [1]. To account for trap states in poly-Si [2] we subtract the trap density, $N_{\mathrm{t}}$, from the net active dopant concentration to determine the effective carrier concentration, $n_{\mathrm{eff}}$ (see Figure 1b). The local resistivity in the $p-i-n$ structure was obtained either from the literature [3-5] or estimated based on the carrier concentration and mobility in poly-Si.

Following the resistivity calculation of each resistor in series from the local $n_{\text {eff }}$, the total shunt resistance, $R_{\text {shunt }}$, was determined by using the expression,

$$
R_{\text {shunt }}=\frac{W_{p}}{2 t} \int_{0}^{\delta} \rho(x) d x
$$

where $\rho(x)$ is the local resistivity, $W_{p}$ is the pitch, and $t$ is the film thickness. In all resistor simulations, $W_{p}$ and $t$ were fixed at $1 \mathrm{~mm}$ and $100 \mathrm{~nm}$, respectively. We calculated the total shunt resistance for different values of isolation region width, $\delta$, over the range of $20-55 \mu \mathrm{m}$ [6-8] and for different dopant diffusion lengths in the poly-Si isolation region, $L_{\text {diff }}=\sqrt{D_{e f f} t}$, where $D_{\text {eff }}$ is the effective diffusivity of the dopant in poly-Si and $t$ is the time over which the diffusion occurs. For diffusion at temperatures $>1000{ }^{\circ} \mathrm{C}$, this corresponds to $D_{\text {eff }} \sim 10^{-10}-10^{-9} \mathrm{~cm}^{2} / \mathrm{s}$ and $t \sim 10^{3} \mathrm{~s}$ to achieve $L_{\text {diff }}$ $\sim 10 \mu \mathrm{m}$. To apply the resistor model more generally for aiding in design of IBC cells, we consider how the width of the region between doped fingers will affect $R_{\text {shunt }}$ across the isolation region. We varied $\delta$ over the range of $20-55 \mu \mathrm{m}$ as $\sim 10-100 \mu \mathrm{m}$ is on the order of commonly used distances between the $p$-and $n$-type fingers of IBCs. $L_{d i f f}$ of the $n$-type tail was varied over the range of $50-100 \mu \mathrm{m}$, corresponding to diffusivities of phosphorus, $D_{P} \sim 10^{-10} \mathrm{~cm}^{-2} / \mathrm{s}$ [9-11] and diffusion times, $t_{\text {diff }} \sim 1 \mathrm{hr}$. For high temperature, $>1000{ }^{\circ} \mathrm{C}$, annealing of $p o l y-\mathrm{Si} / \mathrm{SiO}_{x}$ passivated contacts, these values for $L_{d i f f}$ are reasonable. However, specific values can differ significantly depending on the annealing temperature, poly-Si grain size, and doping level in poly-Si [12-16]. In these simulations, we assume a trap density, $N_{\mathrm{t}}=10^{18} \mathrm{~cm}^{-3}$, in poly-Si. 


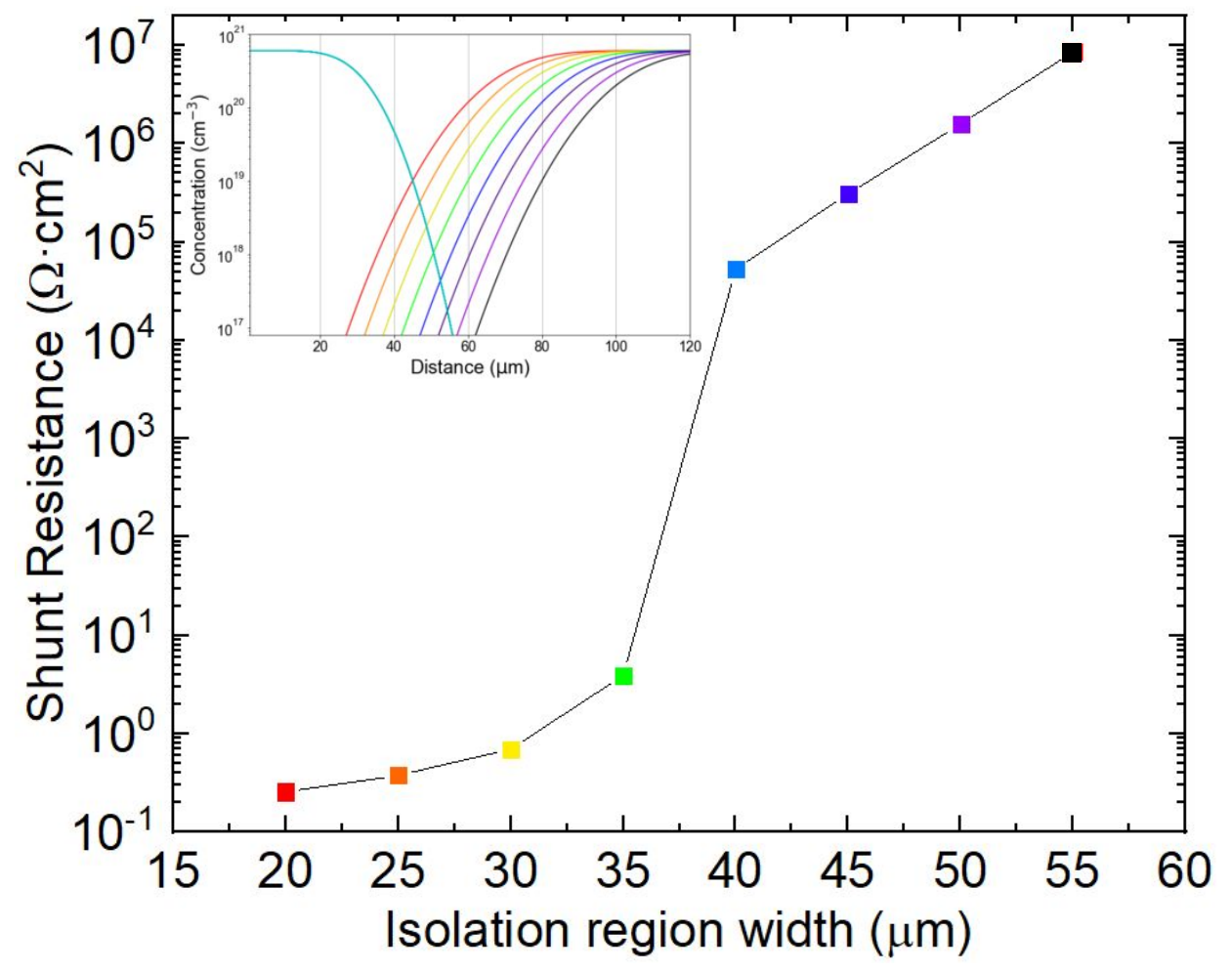

Figure S2 Shunt resistance for different isolation region widths, $\delta$, between $n$ - and $p$-type fingers. The colors of each point correspond to the color for the phosphorus concentration profiles on the right hand side in the inset. The boron concentration profile is shown in cyan on the left side of the inset.

Figure $\mathrm{S} 2$ shows the calculated $R_{\text {shunt }}$ as a function of poly-Si isolation region width for error-function-shaped dopant concentration profiles for the $p$ - and $n$-type doped fingers. These profiles are similar to those that are observed for IBC devices fabricated with ion implantation followed by high-temperature annealing [9, 17]. A large increase in the shunt resistivity from $<1$ to $>10^{5} \Omega \cdot \mathrm{cm}$ occurs for the isolation region widths between $35-40 \mu \mathrm{m}$. As can be seen in the inset of Figure S2, the concentration at the point at which the dopant profiles intersect is less than $N_{\mathrm{t}}$ for isolation region widths, $\delta<40 \mu \mathrm{m}$. Though the net dopant concentration should be negligible right at the single point where the active $p$ - and $n$-type dopants are equal in concentration, the width of the region over which the net dopant concentration is below $N_{\mathrm{t}}$ is much higher when $\delta<40 \mu \mathrm{m}$. This wider region allows for a greater chance that the compensated region will truly isolate the doped regions from one another. 


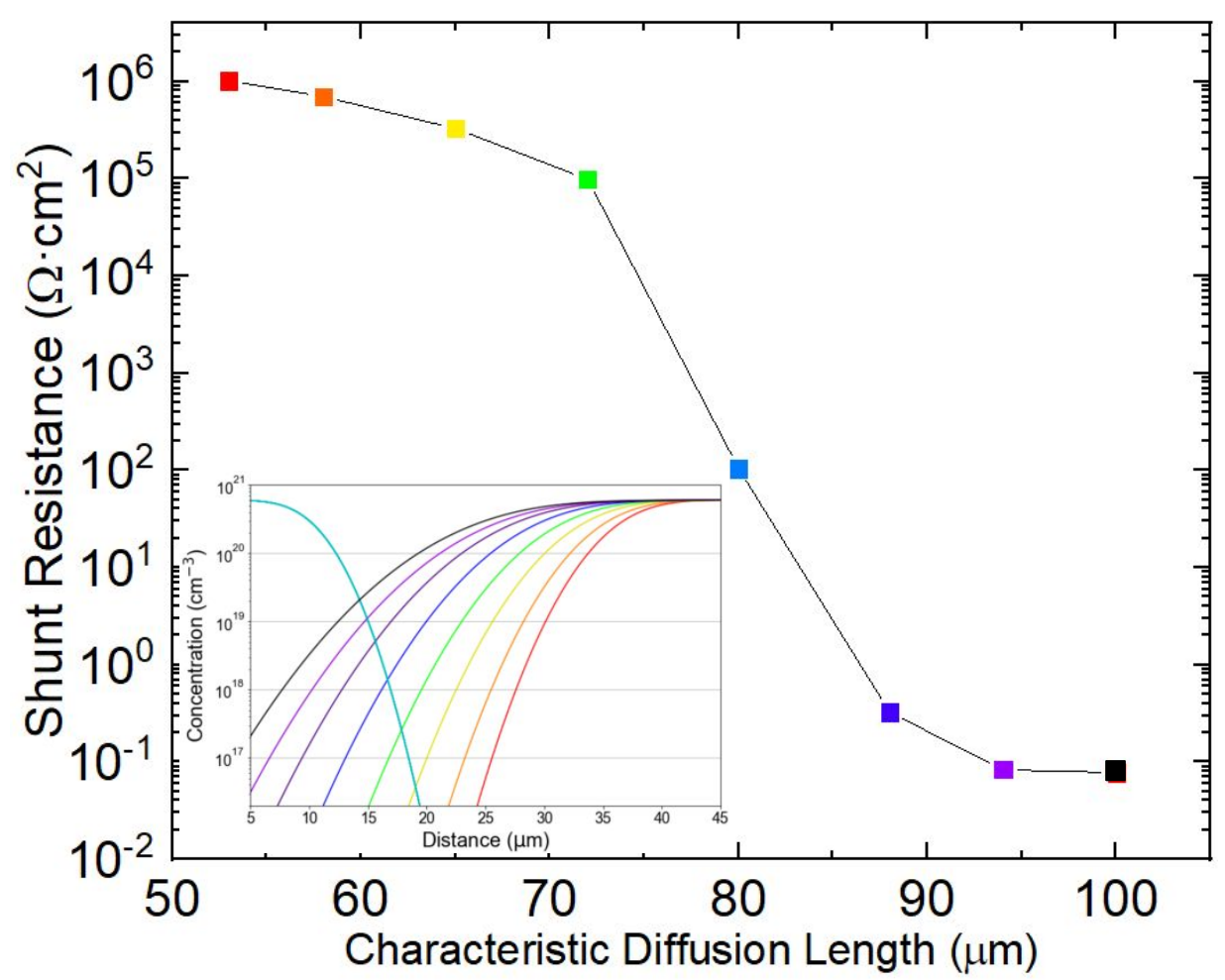

Figure S3 Shunt resistance across the gap region for different $n$-type finger $L_{\text {diff }}$ for constant $p$-type $L_{\text {diff }}$ and constant isolation region width. The inset graph shows a representative overlap between the two dopant profiles, with increasing $n$-type $L_{\text {diff }}$ from the red to the black curve.

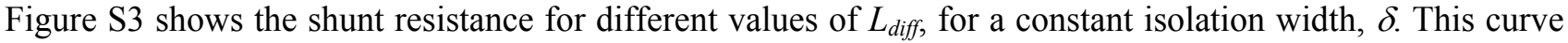
follows a very similar pattern to that seen in Figure S2. Literature values for diffusivity of phosphorus and boron in poly-Si vary by several orders of magnitude depending on the method of formation of poly-Si, dopant concentration, deposition temperature, grain size, and other factors. The diffusivities range from $10^{-15}-10^{-12} \mathrm{~cm}^{2} / \mathrm{s}$ and $10^{-19}-10^{-9}$ $\mathrm{cm}^{2} / \mathrm{s}$ for boron and phosphorus, respectively $[10,12,13,15,18-20]$. These values depend strongly on the poly-Si annealing temperature. Therefore, if a smaller isolation region width, $\delta$, between doped fingers is desired, it may be necessary to reduce process temperature or diffusion time significantly.

\section{$\underline{\text { Simulations of Diode Behavior }}$}

We used error-function profiles with concentrations of $10^{19} \mathrm{~cm}^{-3}$ at the edge of the intentionally defined boundary. These profiles had a characteristic decay length, $L_{\text {diff, }}$ of 125 and $200 \mu \mathrm{m}$ to approximate the profiles measured by TOF-SIMS (see Figure $3 \mathrm{~b}$ in main text). The point of intersection of these concentration profiles is $\sim 6.4 \times 10^{18} \mathrm{~cm}^{-3}$, which is approximately $42 \mu \mathrm{m}$ from the intentionally defined edge of the $p$-type finger. Six discrete trap levels were introduced within the band gap. All equations and parameter values used within the diode simulations using the finite element method are listed below. 


\section{Simulation Details - Parameter Tables}

Table S1: Input parameters for the equations used in finite-element simulations of the $p-i-n$ structure.

\begin{tabular}{|c|c|c|c|}
\hline Parameter & Symbol & Value & Unit \\
\hline Cross-sectional area & $A$ & 1 & $\mathrm{~mm}^{2}$ \\
\hline Temperature & $T$ & 300 & $\mathrm{~K}$ \\
\hline Intrinsic carrier concentration & $n_{\mathrm{i}}$ & $1.5 \times 10^{10}$ & $\mathrm{~cm}^{-3}$ \\
\hline Initial acceptor concentration & $N_{\mathrm{a}}$ & $1 \times 10^{19}$ & $\mathrm{~cm}^{-3}$ \\
\hline Initial donor concentration & $\overline{N_{\mathrm{d}}}$ & $1 \times 10^{19}$ & $\mathrm{~cm}^{-3}$ \\
\hline Width of central gap region & $\overline{W_{\mathrm{g}}}$ & 10 & $\mu \mathrm{m}$ \\
\hline Relative permittivity & $\varepsilon_{0}$ & 11.7 & 1 \\
\hline Band gap & $E_{\mathrm{g}}$ & 1.12 & V \\
\hline Electron affinity & $X_{0}$ & 4.05 & $\mathrm{~V}$ \\
\hline Valence band effective density of states & $\overline{N_{\mathrm{v}}}$ & $1.04 \times 10^{19}$ & $\mathrm{~cm}^{-3}$ \\
\hline Conduction band effective density of states & $N_{\mathrm{c}}$ & $2.8 \times 10^{19}$ & $\mathrm{~cm}^{-3}$ \\
\hline Electron Auger recombination factor & $C_{\mathrm{n}}$ & $2.8 \times 10^{-31}$ & $\mathrm{~cm}^{6} / \mathrm{s}$ \\
\hline Hole Auger recombination factor & $C_{\mathrm{p}}$ & $9.9 \times 10^{-32}$ & $\mathrm{~cm}^{6} / \mathrm{s}$ \\
\hline Hole Capture Cross Section & $\sigma_{\mathrm{p}}$ & $10^{-12}$ & $\mathrm{~cm}^{2}$ \\
\hline Electron Capture Cross Section & $\sigma_{\mathrm{n}}$ & $10^{-12}$ & $\mathrm{~cm}^{2}$ \\
\hline Hole Thermal Velocity & $v_{\text {th,p }}$ & $1.562 \times 10^{7}$ & $\mathrm{~cm} / \mathrm{s}$ \\
\hline Electron Thermal Velocity & $v_{\text {th,n }}$ & $2.042 \times 10^{7}$ & $\mathrm{~cm} / \mathrm{s}$ \\
\hline Direct recombination factor & $C$ & 0 & $\mathrm{~cm}^{3} / \mathrm{s}$ \\
\hline Electron lifetime, SRH & $\tau_{\mathrm{n}}$ & 10 & $\mu \mathrm{s}$ \\
\hline Hole lifetime, SRH & $\overline{\tau_{\mathrm{p}}}$ & 10 & $\mu \mathrm{s}$ \\
\hline Electron mobility & $\mu_{\mathrm{n}}$ & 1450 & $\mathrm{~cm}^{2} /(\mathrm{V} \cdot \mathrm{s})$ \\
\hline Hole mobility & $\mu_{\mathrm{p}}$ & 500 & $\mathrm{~cm}^{2} /(\mathrm{V} \cdot \mathrm{s})$ \\
\hline
\end{tabular}

\section{$\underline{\text { Poisson's Equation }}$}

where,

$$
\nabla \cdot\left(-\varepsilon_{0} \varepsilon_{r} \nabla V\right)=q\left(p-n+N_{d}^{+}-N_{a}^{-}+Q_{t}\right)
$$

$$
Q_{t}= \begin{cases}-q N_{t} f_{t} & , E_{t} \geq E_{i} \\ q N_{t}\left(1-f_{t}\right) & , E_{t}<E_{i}\end{cases}
$$

\section{$\underline{\text { Drift-diffusion Equation }}$}

$$
\begin{aligned}
& J_{n}=q n \mu_{n} \nabla E_{c}+\mu_{n} k_{b} T \nabla n+q n D_{n, t h} \nabla \ln (T) \\
& J_{p}=q p \mu_{p} \nabla E_{v}+\mu_{p} k_{b} T \nabla p+q p D_{p, t h} \nabla \ln (T)
\end{aligned}
$$




\section{Energy levels}

$$
\begin{gathered}
E_{f, 0}=V_{e q}-V_{a} \\
E_{c}=-\left(V+X_{0}\right) \\
E_{v}=-\left(V+X_{0}+E_{g}\right) \\
E_{t}=-\left(V+X_{0}+E_{\text {offset }}\right) \quad \text { where } \quad E_{\text {offset }} \in\{0.275,0.33,0.385,0.715,0.67,0.825\}
\end{gathered}
$$

\section{Trap Occupancy}

\section{Trap-Assisted Recombination}

$$
f_{t}=\frac{1}{1+\frac{1}{g} e^{\left(\frac{E_{t}-E_{f}}{k_{b} T}\right)}}
$$

$$
\begin{aligned}
& N_{t} \frac{\partial f_{t}}{\partial t}=\sigma_{n} v_{t h, n} N_{t}\left(n-n f_{t}-\frac{n_{1}}{g} f_{t}\right)-\sigma_{p} v_{t h, p} N_{t}\left(p f_{t}-g p_{1}\left(1-f_{t}\right)\right) \\
& n_{1}=n_{i} e^{\left(\frac{E_{t}-E_{i}}{k_{b} T}\right) \frac{F_{1 / 2}\left(-\frac{E_{c}-E_{f n}}{k_{b} T}\right)}{e^{\left(-\frac{E_{c}-E_{f n}}{k_{b} T}\right)}}} \\
& p_{1}=n_{i} e^{\left(\frac{E_{i}-E_{t}}{k_{b} T}\right)} \frac{F_{1 / 2}\left(-\frac{E_{f p}-E_{v}}{k_{b} T}\right)}{e^{\left(-\frac{E_{f p}-E_{v}}{k_{b} T}\right)}}
\end{aligned}
$$

\section{$\underline{\text { Auger Recombination }}$}

$$
R_{A}=\left(C_{n} n+C_{p} p\right)\left(n p-n_{i}^{2} \frac{F_{1 / 2}\left(-\frac{E_{f p}-E_{v}}{k_{b} T}\right) F_{1 / 2}\left(-\frac{E_{c}-E_{f n}}{k_{b} T}\right)}{e^{\left(-\frac{E_{f p}-E_{v}}{k_{b} T}\right)} e^{\left(-\frac{E_{c}-E_{f n}}{k_{b} T}\right)}}\right)
$$

\section{Supplementary References}

[1] W. Nemeth et al., "Polycrystalline silicon passivated tunneling contacts for high efficiency silicon solar cells," (in English), Journal of Materials Research, vol. 31, no. 6, pp. 671-681, Mar 28 2016, doi: 10.1557/jmr.2016.77.

[2] T. Kamins, "Hall mobility in chemically deposited polycrystalline silicon," Journal of applied physics, vol. 42, no. 11, pp. 4357-4365, 1971.

[3] B. Tyagi and K. Sen, "On the resistivity of polycrystalline silicon," physica status solidi (a), vol. 80, no. 2, pp. 679-684, 1983.

[4] D. P. Joshi and R. S. Srivastava, "Mobility and carrier concentration in polycrystalline silicon," Solar Cells, vol. 12, no. 3, pp. 337344, 1984/08/01/ 1984, doi: https://doi.org/10.1016/0379-6787(84)90112-1.

[5] A. L. Fripp, "Dependence of resistivity on the doping level of polycrystalline silicon," Journal of Applied Physics, vol. 46, no. 3, pp. 1240-1244, 1975/03/01 1975, doi: 10.1063/1.321687.

[6] C. Hollemann, F. Haase, S. Schäfer, J. Krügener, R. Brendel, and R. Peibst, "26.1\%-efficient POLO-IBC cells: Quantification of electrical and optical loss mechanisms," Progress in Photovoltaics: Research and Applications, 2019, doi: 10.1002/pip.3098.

[7] C. Reichel, R. Müller, F. Feldmann, A. Richter, M. Hermle, and S. W. Glunz, "Influence of the transition region between p- and ntype polycrystalline silicon passivating contacts on the performance of interdigitated back contact silicon solar cells," (in English), Journal of Applied Physics, vol. 122, no. 18, Nov 14 2017, doi: 10.1063/1.5004331.

[8] D. L. Young et al., "Interdigitated Back Passivated Contact (IBPC) Solar Cells Formed by Ion Implantation," IEEE Journal of Photovoltaics, vol. 6, no. 1, pp. 41-47, 2016, doi: 10.1109/jphotov.2015.2483364. 
[9] C. Hollemann et al., "Separating the two polarities of the POLO contacts of an 26.1\%-efficient IBC solar cell," Scientific Reports, vol. 10, no. 1, p. $658,2020 / 01 / 202020$, doi: 10.1038/s41598-019-57310-0.

[10] F. H. M. Spit and H. Bakker, "Diffusion of donor elements (125Sb, 82P, 74(73)As) in polycrystalline silicon," physica status solidi (a), vol. 97, no. 1, pp. 135-142, 1986, doi: 10.1002/pssa.2210970111.

[11] A. D. Buonaquisti, W. Carter, and P. H. Holloway, "Diffusion characteristics of boron and phosphorus in polycrystalline silicon," Thin Solid Films, vol. 100, no. 3, pp. 235-248, 1983/02/18/ 1983, doi: https://doi.org/10.1016/0040-6090(83)90281-X.

[12] D. Losee, J. Lavine, E. Trabka, S. T. Lee, and C. Jarman, "Phosphorus diffusion in polycrystalline silicon," Journal of applied physics, vol. 55 , no. 4 , pp. 1218-1220, 1984.

[13] T. I. Kamins, J. Manoliu, and R. N. Tucker, "Diffusion of Impurities in Polycrystalline Silicon," Journal of Applied Physics, vol. 43, no. 1, pp. 83-91, 1972, doi: 10.1063/1.1660842.

[14] H. Baumgart, H. Leamy, J., G. Celler, K., and L. Trimble, E., "GRAIN BOUNDARY DIFFUSION IN POLYCRYSTALLINE SILICON FILMS ON SiO2," J. Phys. Colloques, vol. 43, no. C1, pp. C1-363-C1-368, 1982. [Online]. Available: https://doi.org/10.1051/jphyscol:1982148.

[15] B. Han et al., "Phosphorus and boron diffusion paths in polycrystalline silicon gate of a trench-type three-dimensional metal-oxidesemiconductor field effect transistor investigated by atom probe tomography," Applied Physics Letters, vol. 107, no. 2, 2015, doi: $10.1063 / 1.4926970$.

[16] M. R. Murti and K. V. Reddy, "Grain boundary diffusion of phosphorus in polycrystalline silicon," Semiconductor Science and Technology, vol. 4, no. 8, pp. 622-625, 1989/08/01 1989, doi: 10.1088/0268-1242/4/8/004.

[17] R. Müller, C. Reichel, J. Benick, and M. Hermle, "Ion Implantation for All-alumina IBC Solar Cells with Floating Emitter," Energy Procedia, vol. 55, pp. 265-271, 2014, doi: 10.1016/j.egypro.2014.08.078

[18] D. J. Coe, "The lateral diffusion of boron in polycrystalline silicon and its influence on the fabrication of sub-micron MOSTs," SolidState Electronics, vol. 20, no. 12, pp. 985-992, 1977/12/01/ 1977, doi: https://doi.org/10.1016/0038-1101(77)90208-8.

[19] I. C. Post and P. Ashburn, "Investigation of boron diffusion in polysilicon and its application to the design of pnp polysilicon emitter bipolar transistors with shallow emitter junctions," IEEE transactions on electron devices, vol. 38, no. 11, pp. 2442-2451, 1991.

[20] S. Nédélec, D. Mathiot, and M. Gauneau, "Diffusion of boron in polycrystalline silicon," in ESSDERC'96: Proceedings of the 26th European Solid State Device Research Conference, 1996: IEEE, pp. 153-156. 